\title{
LE MODÈLE \\ MATHÉMATIQUE DU DELTA \\ DU MÉKONG
}

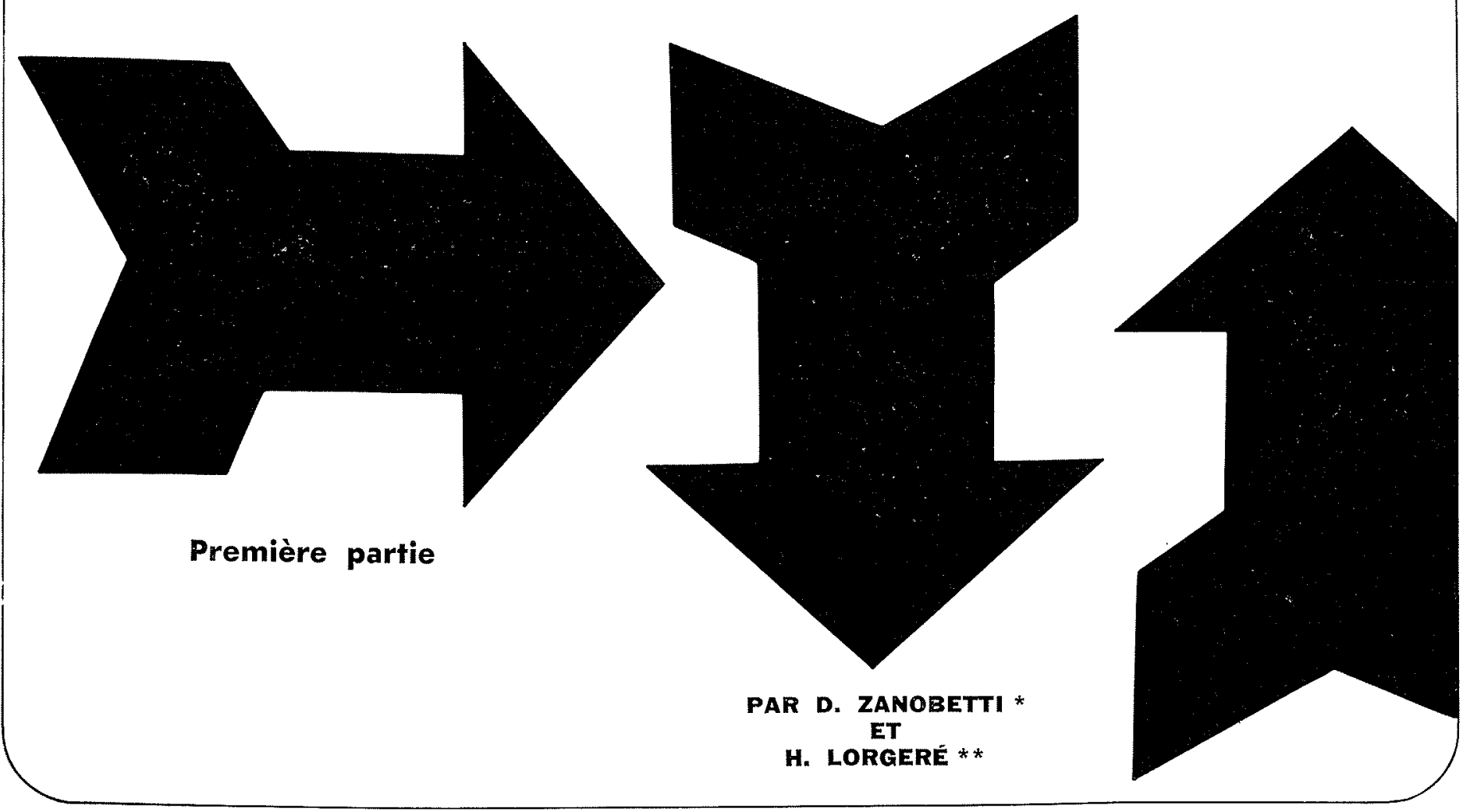

\section{Introduction et aperçu du projet}

\subsection{Introduction.}

Le delta du Mékong constitue une entité hydraulique dont l'ampleur et la complexité s'imposent à l'échelle mondiale.

Habité par dix millions de Cambodgiens et de Vietnamiens, il est le siège de deux des grandes civilisations deltaïques du monde et, de tous temps, l'ingéniosité et le labeur de l'homme se sont efforcés, d'un côté, de s'adapter au mieux aux conditions naturelles, de l'autre, de modifier la nature par des améliorations à but immédiat.

A l'heure actuelle, il devient possible de concevoir, pour le delta et pour la région plus vaste de tout le bassin inférieur du grand fleuve, l'aménagement de grands ensembles.

Cet aménagement du Mékong a précisément pour objet la mise en valeur intégrée des ressources hydrauliques du Bassin inférieur du Mékong, tant sur le cours principal que sur les affluents, en matière de production d'énergie hydroélectrique,

* Professeur à l'Université de Bologne, ancien directeur de la Division des etudes, de la recherche et de l'enseignement technique de l'Organisation des Nations Unies pour l'Education, la Science et la Culture (UNESCO) et directeur du projet du modèle mathématique du Delta du Mékong;

* Chef adjoint du Service hydroélectrique à la Socièté Grenobloise d'Etudes et d'Applications Hydrauliques (SOGREAH) de Grenoble et ingénieur en chef du projet du modèle mathématique du Delta du Mékong. d'irrigation, de maîtrise des eaux, d'aménagement de bassins versants, d'adduction d'eau ainsi qu'en d'autres domaines connexes, pour le bien de toutes les populations du Bassin, sans distinction de nationalité, de religion ou de politique.

Ce projet du Mékong est dirigé par le Comité pour la coordination des études sur le Bassin inférieur du Mékong, créé en 1957 par les Gouvernements du Cambodge, du Laos, de la Thaïlande et de la République du Viet-Nam, comme suite à une recommandation adoptée par la CEAEO à sa treizième session, tenue en mars de cette année.

Le but général de ces travaux n'est autre que la réalisation des objectifs envisagés dans le rapport de la Mission de l'Assistance technique des NationsUnies, dirigée par le général Raymond Wheeler : «Programme d'études et d'enquêtes pour l'intégrale mise en valeur du Bassin inférieur du Mékong», TAA/AFE/3, janvier 1958.

La réalisation de cet objectif implique une gamme d'activités très étendue, comprenant notamment la mise au point d'un programme de développement intégré du bassin ainsi que l'étude, la construction, le financement et la gestion des différents aménagements.

Le but du texte ci-après est de rendre compte d'une de ces études, fondamentale parce que préalable à beaucoup d'autres : le modèle mathématique du Delta.

L'étude, proposée en 1960 par le Comité de coordination, financée, comme on le verra, en grande partie par le Fonds spécial des Nations-Unies, fut confiée pour exécution en 1961 à l'Organisation des 
Nations-Unies pour l'Education, la Science et la Culture (UNESCO). Celle-ci, maître d'œuvre, fit un appel d'offres international à la suite duquel les campagnes de mesures et l'étude propre du modèle furent attribués à la Société Grenobloise d'Etudes et d'Applications Hydrauliques (SOGREAH, France).

Les travaux, commencés en 1962, se sont terminés en 1966: ils ont été décrits en détail en un rapport final soumis aux Etats riverains et aux organisations des Nations-Unies concernés. [Voir bibliographie en fin de cel article.]

L'étude a constitué, par l'ampleur de la région et par sa complexité hydraulique, par la multitude des facteurs pris en compte, l'exemple de modèle mathématique hydraulique le plus important à notre connaissance et il a paru utile, avec la permission de la Direction Générale de l'UNESCO, de la résumer dans cet article.

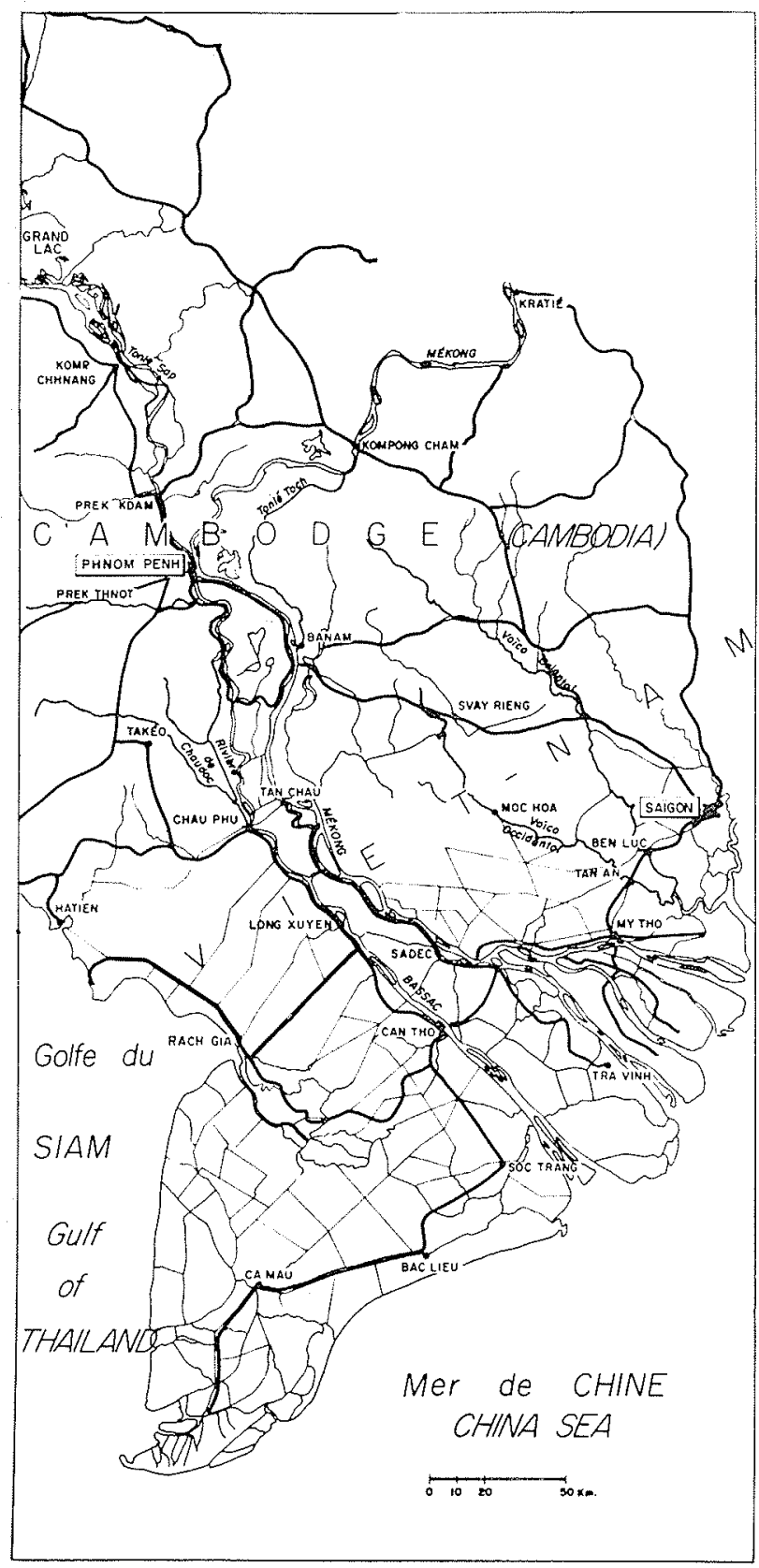

A/ Delta du Mékong / Mekong Delta.
Après une description sommaire du projet de barrage du Tonlé Sap qui est à l'origine du modèle, nous traiterons donc successivement dans les paragraphes suivants :

2. des campagnes de mesures pour l'oblention des données du modèle;

3. de la physionomie ou des caractéristiques du modele mathématique el la comparaison avec l'alternative du modèle physique;

4. des principes du calcul du modèle mathématique, son analyse et sa programmation;

5 . de la construction du modèle mathématique;

6. de son réglage;

7. de l'exploitation pour l'étude de l'influence du barrage du Tonlé Sap.

Nous donnerons enfin quelques informations sur la bibliographie, sur le coût de l'étude, sur les personnes qui y ont participé.

\subsection{Le projet de barrage du Tonlé Sap.}

(Cf. ECAFE. Comité du Mékong. Contrôle des crues, $n^{\circ} 12$. Mise en valeur des ressources hydrauliques du Bassin inférieur du Mékong (page 55/60). Bangkok, février 1957.)

La capitale du Cambodge, Phnom Penh, est un carrefour hydraulique. Quatre rivièes y parviennent :

- le Mékong amont, qui véhicule la crue de base venant du nord;

- le Mékong aval et le Bassac qui en transportent une grande partie jusqu’à la mer de Chine;

- le Tonlé Sap qui remplit avec une autre partie l'énorme réservoir naturel du Grand Lac (72 milliards de mètres cubes) et le vide en décrue vers l'aval, c'est-à-dire vers le sud.

Comme ces trois défluents de la crue de base: Mékong aval, Bassac, Tonlé Sap sont insuffisants, le reste de la crue, qu'ils n'ont pu transporter, inonde la plaine du Delta.

Bien que l'on voie l'importance de la régularisation naturelle, elle reste encore insuffisante:

- pour certaines crues trop puissantes ou trop rapides, qui causent des dommages à l'agriculture, aux routes, aux habitations;

- pour certaines décrues trop rapides, préjudiciables à la navigation et à l'agriculture (avance du front salin).

Pour pallier ces inconvénients, on a pensé à une modulation artificielle de l'immense réservoir naturel du Grand Lac, en interposant un barrage de régulation, au site de Kompong Chnnang, sur le Tonlé Sap qui le relie au Méliong.

Sa fermeture, en début de crue, éviterait que le Lac ne soit encombré par la montée préalable et inoffensive des eaux; lorsque la crue commencerait à devenir critique, on ouvrirait alors le barrage, absorbant ainsi la force de la pointe sauvage, dans la réserve maintenue intacte dans le Lac.

Inversement, une vidange commandée par le barrage, permettrait d'espérer une soudure plus harmonieuse avec le début de la crue suivante et la régularisation de la décrue. 
Cette double régularisation a pour but:

- en crue :

- de diminuer la hauteur maximale atteinte par les crues,

- de réduire la vitesse de montée des caux, qui peut atteindre une valeur néfaste pour l'agriculture (plus de $10 \mathrm{~cm} /$ jour);

- en décrue :

- de régulariser le régime de décrue dans le Delta :

- du point de vue navigation dans le Mékong (élévation du niveau de l'eau),

- du point de vue agriculture:

- permetre une irrigation de saison sèche,

- faciliter la soudure avec la saison humide suivante,

- commander le régime de descente des eaux.

Enfin, celte amélioration hydraulique s'accompagne de conséquences importantes qui intéressent des spécialistes tels que:

- ichtyologues : possibilités d'augmenter la richesse piscicole du Grand Lac, naturellement déjà importante;

- agronomes : possibilités d'étendre les cultures de saison sèche, d'introduire de nouvelles pratiques culturales et de nouvelles variétés.

Pour pouvoir juger l'influence du barrage $d u$ Tonlé Sap, il fallait toutefois connaittre la propagation naturelle des crues (sans barrage), puis étudier la propagation artificielle des crues avec barrage.

Il fallait donc un modèle hydraulique, et c'est justement en considération de l'ampleur géographique de ce problème, de l'énorme amplitude des paramètres hydrauliques, de la multitude des résultats à donner que les experts ont choisi, comme modèle hydraulique, un modèle mathématique.

Le projet de ce modèle mathématique a été divisé en quatre phases :

a) rassemblement des données et reconnaissance du terrain;

b) exécution d'un modèle préliminaire;

c) campagnes de mesures;

d) exécution du modèle définitif.

\section{Phase a) - Reconnaissances :}

Les archives des Services publics sont très fournies dans les deux territoires; la mission consistait $\grave{a}$ en prendre connaissance et à choisir ce qui était intéressant pour le modèle mathématique.

En outre, la reconnaissance sur place permettait de se familiariser avec l'hydranlicue du Delta et de prendre contact avec les autorités locales et internationales intéressées par le projet.

\section{Phase b) - Modele préliminaire :}

Ce modèle correspondait à plusieurs buts :

1. Mettre au point une méthode nouvelle : le modèle mathématique, appliqué à un écoulement à deux dimensions.

En effet, pendant la crue, l'inondation forme une nappe continue qui recouve la plaine; la hauteur d'eau et la vitesse varient avec le point considéré, c'est-à-dire avec ses deux coordonnées. Or, le modèle mathématique n'avait été utilisé qu'à l'occasion d'écoulements uni-dimensionnels (rivières, canaux).

\section{Préciser la campagne de mesures:}

Les données des archives étaient insuffisantes pour le modèle mathématique; il fallait faire un choix judicieux des mesures complémentaires, afin d'orienter la campagne de mesures et d'en obtenir un rendement. maximal.

\section{Connaitre l'hydraulique du Della:}

L'exécution du modèle préliminaire a en efiet permis de voir les points sensibles et importants de l'écoulement: en passant ainsi en revue tout le Delta, toute son hydraulique a été étudiée par la mème occasion.

Ce modèle préliminaire étail donc utile en Iuimême.

\section{Faciliter le réglage définitif.}

Pour le réglage du modèle préliminaire, le Delta avait été divisé en plusieurs parties, car la connaissance des archives était variable suivant les régions : on avait fait un découpage correspondant à des degrés de connaissance équivalents; en outre, comme dans les archives, on n'avait pas d'année unique qui ait été suivie dans l'ensemble du Delta, chaque morceau était réglé sur une crue différente; enfin, il portait sur deux mois de crue environ, au maximum, et il s'agissait de crues assez anciennes (1934 à 1942).

Le réglage du modèle définitif a porté, lui :

- sur l'ensemble du Delta,

-..- sur deux crues completes, 1963 et 1964, d'allure

très différente,

- sur cing mois,

et cependant, d'emblée, le réglage du modèle définitif a obtenu les résultats suivants sur la crue de 1963: $59 \%$ des 300 points de calcul du Delta représentaient la réalité à moins de 20 centimètres près.

L’étude préliminaire a donc facilité le réglage définitif.

\section{Phase c) - Campagne de mesures :}

Initialement, une seule campagne était prévue pour 1963: compte tenu des délais d'installations et d'acheminement du matériel, du démarrage des opérations (mai 1962), il fallait être prêt le $1^{\mathrm{er}}$ juin 1963.

Donc, au vu des archives, les dimensions générales de la campagne de mesures avaient été définies dès septembre 1962; et au fur et à mesure des résultats du modele préliminaire, des orientations successives ont donné la forme définitive de la campagne de mesures.

La crue 1963 a été une crue faible : comme l'exploitation du barrage (et par suite du modèle) était intéressante pour les hauts niveaux, le réglage sur la crue 1963 était insuffisant.

On a donc décidé de poursuivre une deuxième campagne au cours de l'année 1964: la crue a été juste médiane; toutefois, son allure très brutale a été très différente de la crue progressive de 1963.

Il a été à nouveau décidé de faire une troisième 


\section{ZANOBETTI et H. LORGERE}

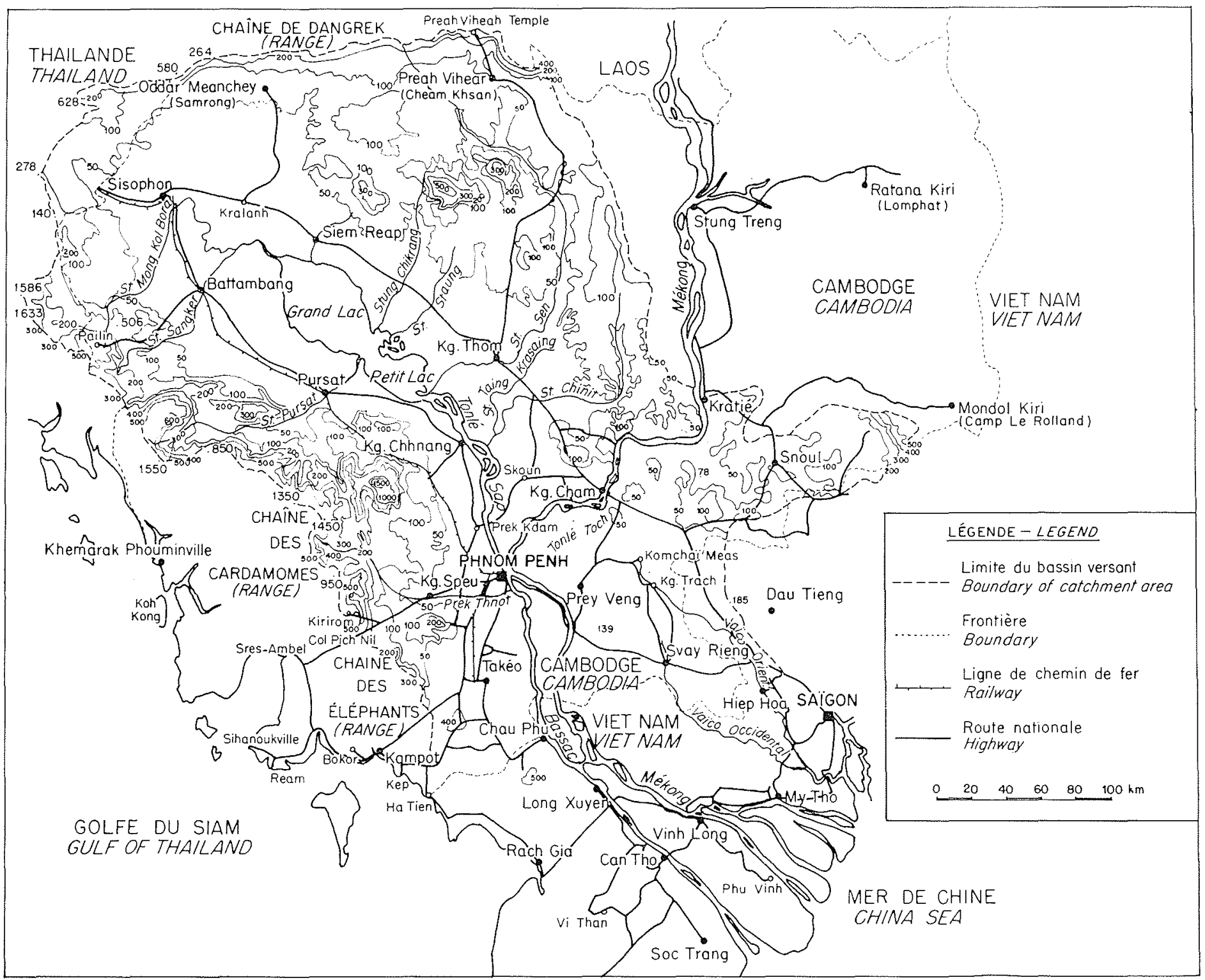

B/ Carte hypsométrique du Delta du Mélkong / Hypsometrical map of Melong Delta.

campagne de mesures 1965: la crue 1965 a été une des plus basses connues et la campagne a pris fin en octobre 1965. Cette crue n'a pas été utilisée pour le réglage du modèle définitif.

Il reste, dans les deux territoires, toute une infrastructure limnigraphique et limnimétrique, que les gouvernements riverains utiliseront.

\section{Phase d) - Modèle définitif :}

Le modèle définitif a donc été réglé sur l'ensemble des deux crues 1963, 1964; bien qu'elles aient en pratiquement la même puissance, leur forme différait totalement : 1964 très brutale, 1963 progressive.

La crue très forte de 1961 a été utilisée sur le modèle, mais elle n'a pu être réglée avec la même précision que les précédentes, car les données sont beaucoup moins nombreuses qu'en 1963 et 1964 .

De même, la décrue type 1963-1964, nécessaire pour l'étude de la régularisation en vidange, a fait l'objet d'un réglage particulier.

En finale, le modèle définitif a été réglé sur les deux crues 1963, 1964 et la décrue type 1963-1964.
On a pu alors procéder à l'exploitation du modèle, c'est-à-dire :

Le modèle réglé reproduit la nature et sert de référence par la suite; on introduit le barrage par sa loi de fonctionnement.

Sur les trois crues précédentes 1961, 1963, 1964, on en observe la répercussion.

Ensuite a lieu l'interprétation des divers résultats, travail important et considérable.

\section{Campagnes de mesures}

\subsection{Récapitulatif des campagnes de mesures.}

Trois crues successives ont été mesurées, dans tout le Delta : 1963, 1964, 1965.

Le tableau de la page suivante rassemble les caractéristiques numériques de ces trois campagnes, et les paragraphes postérieurs à ce tableau donnent quelques informations plus détaillées. 


\section{Topographie :}

Nivellement de précision ( $\mathrm{km}$ ). (Cambodge 1720 Vietnam $\frac{700}{2420}$

Hydrographie :

Nomrre

Sections en travers, orifices rou- (Cambodge 330 tiers. . . ............ Vietnam $\quad 230$ 560

Détermination de la capacité du Grand Lac:

56 heures de survol aérien;

$220 \mathrm{~km}$ de nivellements.

Stations limnimétriques : équipement et exploitation :

_ échelles. .............. Clambodge 127

Vietnam $\quad 72$

199

- limnigraphes à bulle.......

\begin{tabular}{lr} 
Vietnam & 14 \\
\hline
\end{tabular}

- limnigraphes à flotteur..... $\begin{array}{ll}\text { Cambodge } & 2 \\ \text { Vietnam } & 1\end{array}$
Mesures de débit. Cambodge

630

Vietnam $\frac{598}{1228}$

Mesures de salinité.......... Vietnam 3596

Mesures de turbidité.......... Cambodge 520 Vietnam 208

Stations météorologiques fonda- $\begin{array}{r}\text { Cambodge } 1 \\ \text { (Prey Veng) }\end{array}$ mentales. ............. Vietnam 1

Kilométrage des véhicules (km) Cambodge 387000 634000

Heures de marche : embarca-

tions............... (h) / Cambodge 1340 Vietnam -1

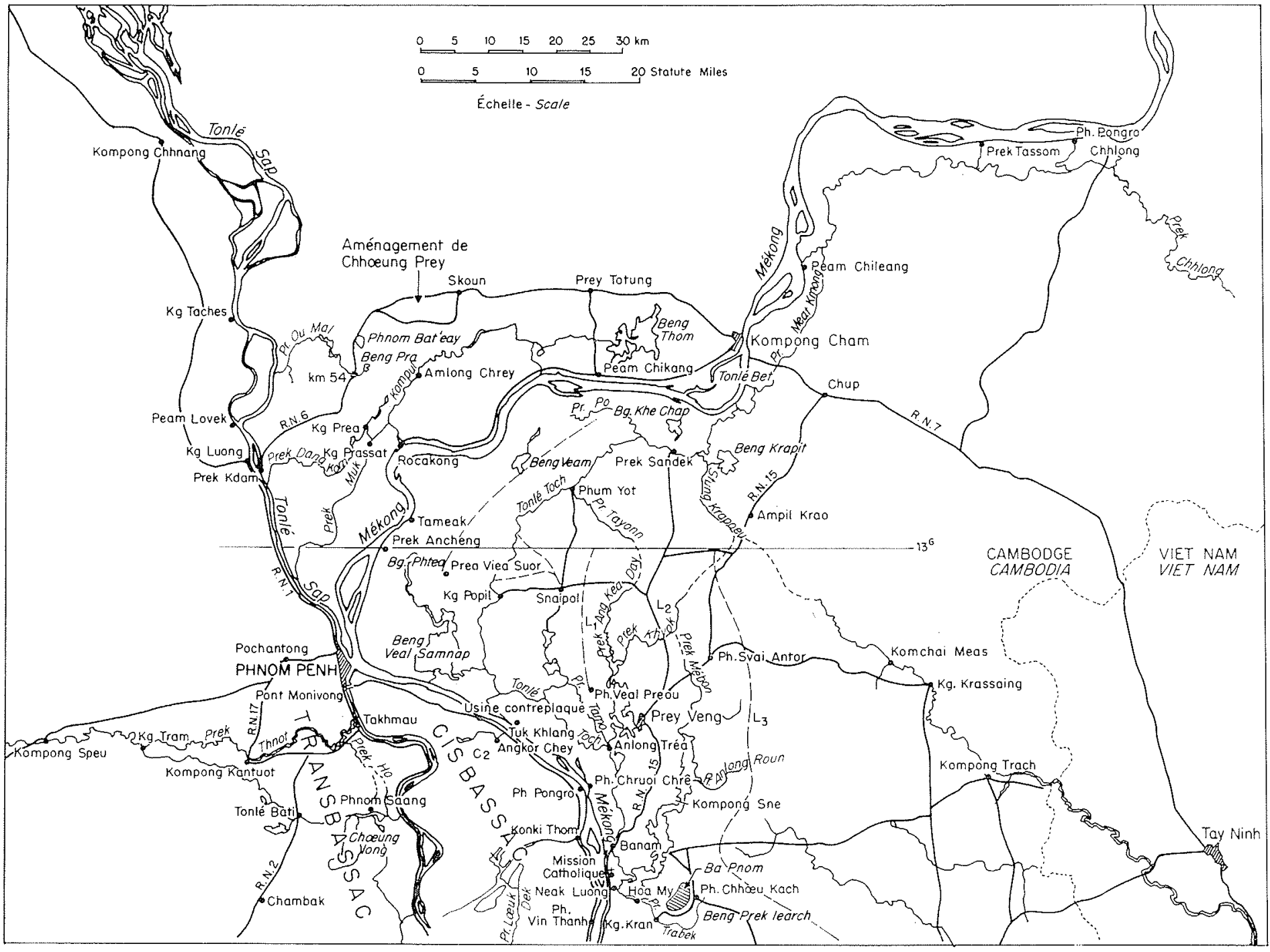

C/ Carte d'ensemble. Cambodge. General map, Cambodia. 


\subsection{Topographie.}

Les $2420 \mathrm{~km}$ ont été principalement faits, pour le rattachement des stations limnimétriques au nivellement général $(220 \mathrm{~km}$ ont servi à la détermination de la capacité du Grand Lac).

Il y a eu 199 stations au total, soit une moyenne de $11 \mathrm{~km}$ de nivellement par station.

La précision est un excellent deuxième ordre, même un bon premier ordre par endroits.

L'importance de la cohérence du nivellement a été démontrée une fois encore; l'étude comparée des résultats récents et des observations anciennes a permis de relever un hiatus de nivellement: $1,16 \mathrm{~m}$ sur l'échelle fondamentale de Phnom Penh Port (noter que Phnom Penh est à la cote $+12 \mathrm{~m}$ et à $330 \mathrm{~km}$ de la mer de Chine).

Le modèle mathématique préliminaire, première image de l'ensemble de l'écoulement, arait mis en doute les observations de certaines échelles; il avait pu même donner le sens et l'ordre de grandeur de l'anomalie; dans tous les cas, les vérifications directes ont confirmé l'erreur attendue.

Le modèle mathématique avait besoin d'une carte hypsométrique du Delta, dont l'équidistance des courbes de niveau fût au moins le mètre; elle n'existait pas.

Au cours d'un travail cartographique important, il a été effectué la synthèse de tous les relevés locaux, de toutes les cartes existantes et on les a rapportés au même système de nivellement. Cet énorme travail couvre 25 feuilles au $100000^{\circ}$, soit $25 \times 1850=46260 \mathrm{~km}^{2}$.

\subsection{Hydrographie.}

La topographie des rivières est fondamentale, puisqu'elle définit les conditions limites aux parois.
Les sections mesurées vont de $18 \mathrm{~m}^{2}$ jusqu'à $20800 \mathrm{~m}^{2}$. On a procédé par ultrasons, sondage direct.

Leur rattachement au nivellement s'est fait par le plan d'eau, dans la plupart des cas.

Les profils en long des rivières font apparaître des structures particulières :

A u Cambodge, le Mékong, le Bassac, le Tonlé Sap sont bordés par des cuvettes, souvent en chaînes; et précisément certains by-pass naturels, importants, (Muk Kompul, Tonlé Toch) correspondent, semble-t-il, à d'anciens chapelets de cuvettes, dont les seuils séparateurs ont été rabotés par les crues successives.

En effet, leurs profils en long sont constitués par des biefs successifs, séparés par des hants fonds, utilisés aux basses eaux comme gués ou assises de barrages à poissons : certaines agglomérations, situées à l'extrémité amont des très longs biefs continus aval, constituent les points limites de la navigation permanente: des ports fluviaux (Kompong Popil, Kompong Prés, Kompong Thom) s'y sont d'ailleurs installés.

A $u$ Vietnam, les canaux artificiels ou naturels rachs inondent, puis draînent la plaine; le profil en long de certains canaux montre souvent un dos d'âne en leur milieu : les marées différentes, à chaque embouchure du canal, interfèrent au milieu où elles créent une eau morte qui favorise les dépôts. Il y correspond d'ailleurs généralement un port où les navigants attendent la direction favorable de la marée descendante.

Lorsque cette nappe continue traverse un drain, naturel ou non, elle lui cède une partie de son débit el le restant suit la pente générale; c'est donc une nappe progressivement appauvrie, qui finit par aboutir à la mer, directement ou non.

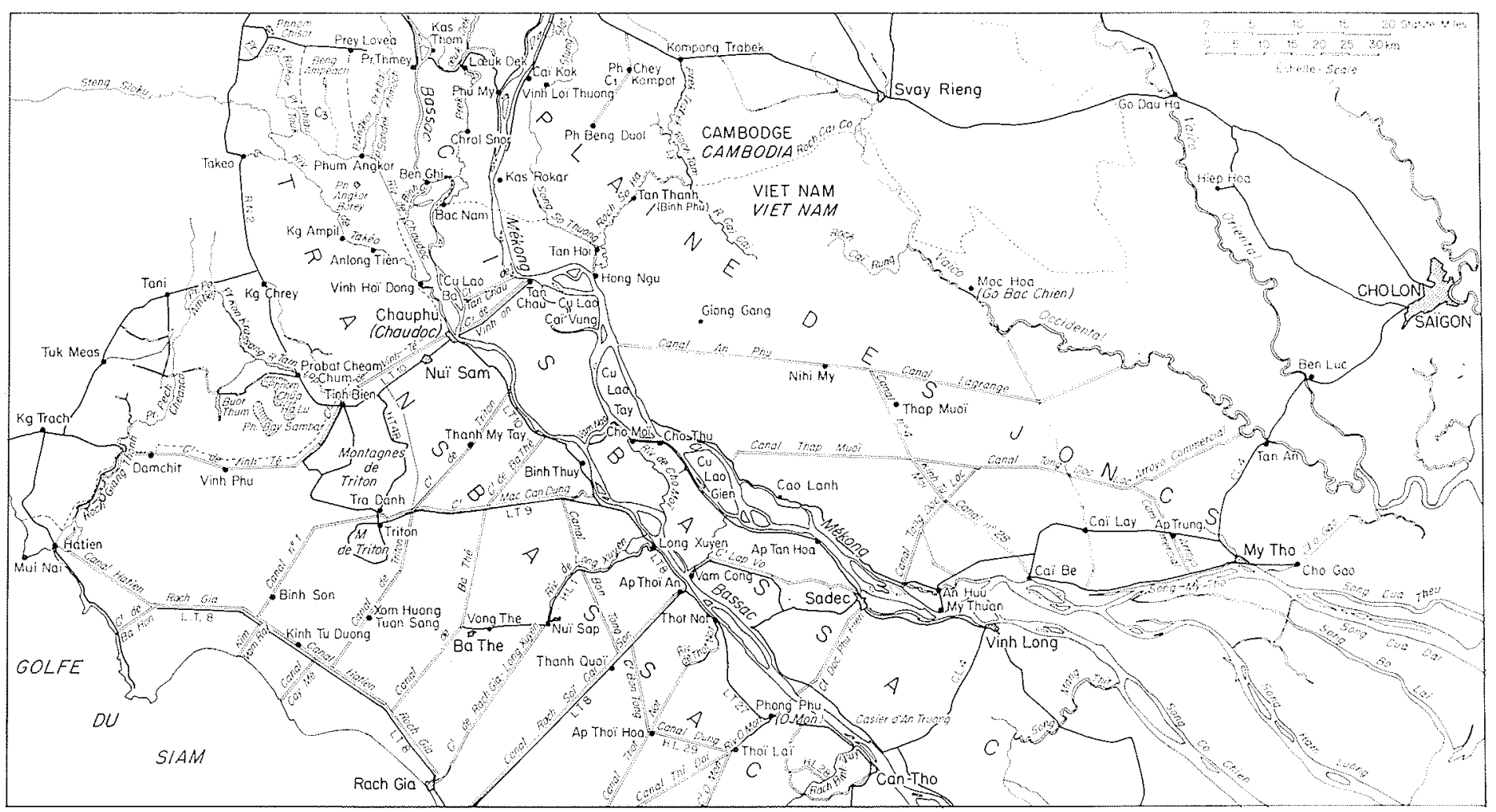

D/ Carte d'ensemble. Cambodge-Vietnam. General map. Cambodia-Vietnam. 
Ces drains recueillent donc, tout au long de leur cours, un sur-débit perpétuel, leur débit va croissant; comme la pente générale est imposée, leur section monillée va rapidement en croissant, d'amont en aval; cette croissance rapide s'explique par l'eflet de va-et-vient de la marée, et par la progression vers l'aval de la finesse des fonds, qui s'accompagne d'une diminution de la pente motrice.

Les relevés des profils montrent en effet cette rapide augmentation des sections à l'aval :

Le Mékong, lui-même, à l'aval de Phnom Penh, a des fonds à :

$5,50 \mathrm{~m}$ pour une largeur de $1500 \mathrm{~m}$; au droit de Mythuan, le fond est à - $35 \mathrm{~m}$ pour une largeur de $800 \mathrm{~m}$.

Le lit du Bassac, à Phnom Penh, émerge aux basses eaux; par contre, sa section mouillée à l'embouchure a les dimensions de celle du Mékong.

La rivière de Takéo, à l'amont, a une section de quelques dizaines de mètres carrés; elle a $2000 \mathrm{~m}^{2}$ à l'aval, au droit de Chauphu.

Le Tonlé Toch, à son extrémité nord, a son fond à $+0,40 \mathrm{~m}$, sa largeur est à $74 \mathrm{~m}$; à son débouché sud, au droit de Banam, son fond est à $-5 \mathrm{~m}$ et sa largeur est de $125 \mathrm{~m}$.

\subsection{Détermination de la capacité du Grand Lac.}

Ce Grand Lac est le réservoir naturel qui régularise la crue annuelle du Mékong: le niveau du Lac varie, en moyenne de $9 \mathrm{~m}$.

Le fonctionnement du barrage sur le 'Tonlé Sap changera le régime du Lac; pour que l'exploitation de cette retenue naturelle puisse être alors définie, il fallait connaître la capacité du I.ac.

On l'a longtemps ignorée, car les rives du Lac sont plates, marécagenses, encombrées de végétation; et le nivellement de référence autour du Lac n'a été fait que récemment.

En attendant, diverses estimations avaient été faites, aboutissant à des chiffres très divergents: pour la cote +11 , on passait de 34 milliards à 79 milliards de mètres cubes.

On a donc inclus, dans le projet, une détermination de la loi niveau/surface/volume du Iaac.

Au cours de l'année 1963, deux moyens ont été utilisés concurremment :

$1^{\circ}$ Survols aériens,à des époques différentes, qui permeltaient de repérer le contour des eaux du Lac; ce contour matérialise en effel, sur le terrain naturel, une courbe de niveau, dont la cote est donnée par les échelles et les limnigraphes du Lac.

Ce procédé a pu être employé jusqu'au niveau maximal de la crue 1963 ; comme il était inférieur au maximum maximorum, la courbe de capacité avait besoin d'un complément d'information, qui a nécessairement été eflectué par un procédé différent du premier.

\section{C'est-à-dire :}

$2^{\circ}$ Antennes de nivellement effectuces à partir de la route périphérique au Lac.

Bien que les deux déterminations diffèrent, dans leurs principes, le point correspondant à la détermination topographique s'est placé, sans difficulté, dans l'extrapolation de la courbe définie par les survols aériens.

Les deux méthodes se sont confirmées mutuellement.

La capacité du Lac est de :

$$
0 \mathrm{~m}^{3} \text { à la cote }+0,60 \mathrm{~m} \text { NGK }
$$

72 milliards de $m^{3}$ à la cote +11 m NGK

La valeur médiane des niveaux atteints, d'environ $9 \mathrm{~m}$, correspond à une capacité de 48 milliards de $\mathrm{m}^{3}$.

Ce volume est rempli :

- par le Mékong, au travers du Tonlé Sap et de la plaine inondée au sud du Lac;

- par le bassin versant propre du Grand Lac, qui couvre $44000 \mathrm{~km}^{2}$.

Le modèle mathématique et les campagnes de mesures 1963 et 1964 ont montré que la proportion due au bassin versant est de :

$$
\begin{aligned}
& 18,7 \% \text { en } 1963 \text {, } \\
& 22,9 \% \text { en } 1964 ;
\end{aligned}
$$

soit en moyenne $20,8 \%$.

Ces valeurs ont été déduites, à partir des débits provenant du Mékong, et de la capacité du Lac, par bilan hydraulique, au moyen du modèle mathématique.

Elles englobent la pluie et l'évaporation, sur le Lac lui-même.

On peut lui comparer la valeur donnée par la campagne hydrologique, effectuée par les missions françaises en 1962-1963 autour du Grand Lac (pour la même région, c'est-à-dire non compris les affluents au sud de la ligne Ponley-Snoctrou-Kompong Thom; Stung Sen exclus).

Ces mesures directes donnent une proportion de $27,8 \%$ (l'évaporation et la pluie du Grand Lac comprises). Quand on sait la difficulté des mesures en hydrologie, on peut s'estimer très satisfait de la concordance des trois valeurs.

En conclusion, en moyenne, $24 \%$ de la capacité du Lac sont remplis par son propre bassin versant et il n'en reste que $76 \%$ pour régulariser la crue du Mékong. Inversement, la vidange du Lac bénéficie de son bassin versant, fraction non négligeable.

\subsection{Réseaux et mesures limnimétriques.}

Le réseau limnimétrique comprenait 22 stations, dans le Delta du Mékong, avant le démarrage des campagnes U.N.E.S.C.O.

Ces 22 stations étaient localisées :

- le long des axes hydrauliques principaux: Tonlé Sap, Mékong, Bassac;

- le long du front de mer ouest du Vietnam.

Or, le modèle mathématique doit représenter la nappe d'inondation qui couvre le Delta, y compris les axes hydrauliques principaux.

En conséquence, les stations initiales ont été : - d'une part, multipliées sur les axes principaux où leur densité initiale était trop faible pour les besoins du projet;

- d'autre part, installées sur des axes secondaires 
qui n’étaient pas on plus observés : Tonlé Toch, Muk Kompul; rivières de Loeuk Dek, de Chaudoc, de Takéo; Vam Nao; rivières de Chomoï; canal Caï San...

- enfin, réparties dans la zone inondée, sur les rives de drains secondaires ou au bord de cuvettes importantes.

Le nombre des stations a été ainsi porté de 22 à 221 .

Au début, les limnigraphes à bulle étaient répartis dans les deux territoires; mais leur utilisation s'est avérée bien plus opportune au Vietnam (marée), où on a augmenté le nombre.

Deux limnigraphes à flotteur ont été installés dans le Grand Lac : un au centre de sa partie sud; l'autre au nord, au large du débouché de la rivière de Siem Réap.

Ils ont demandé la construction d'une superstructure en bois, élevée et difficile : leur exploitation a été délicate (tempêtes sur le Lac, isolement géographique).

Un troisième limnigraphe a été installé sur le canal de Bahon, dans le Trans Bassac Vietnamien, au Vietnam : l'exploitation en est assurée par le personnel de la Cimenterie d'Hâtien.

Les mois principaux de crue dans le Delta sont: août, septembre, octobre, novembre.

La reconnaissance sur le terrain, l'installation, le nivellement ont commencé à la mi-décembre 1962 et ont été terminés d'abord au Cambodge, où la crue se fait sentir en premier.
A $u$ Cambodge, le 1er mai 1963, 72 stations sur 127 étaient en service.

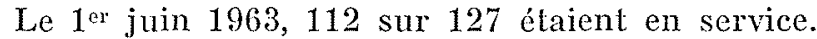
La dermière installation a eu lieu le 23 juillet 1963.

A $u$ Vietnam, 26 stations sur 72 étaient en service le $1^{\mathrm{er}}$ mai 1963.

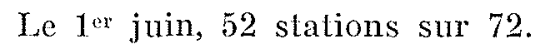

La dernière échelle a été installée le 19 juillet 1963.

En moins de 6 mois, 164 stations sur 199 étaient installées et fonclionnaient avec le personnel convenable, et tout le réseau était prêt pour l'époque de la crue proprement dite (août, ..., novembre).

Les enregistrements de crue ont évidemment permis de suivre la genèse de la propagation de la crue.

Tandis que l'amplitude annuelle des niveaux à l'entrée du Delta ( $\mathrm{Kg}$ Cham) a atteint $13 \mathrm{~m}$, elle se réduit à un peu plus de $1 \mathrm{~m}$ dans l'extrême aval du Mékong.

Au Cambodge, l'écoulement initial est contenu dans le Mélkong; mais, avec la crue, l'eau inonde les cuvettes Iatérales par des brêches, naturelles ou non, au travers des bourrelets de berge; ces cuvettes indépendantes au début, finissent, avec le développement de la crue, par communiquer et constituer une nappe continue. Cette nappe suit alors lentement le sens de l'écoulement général imposé par les grands axes hydrauliques : la plaine devient le lit majeur du fleuve, qui finit par recouvrir sous les eaux les bourrelets de berge. Car le Mékong et
E/ Hydrogrammes des crues. Flood hydrographs.

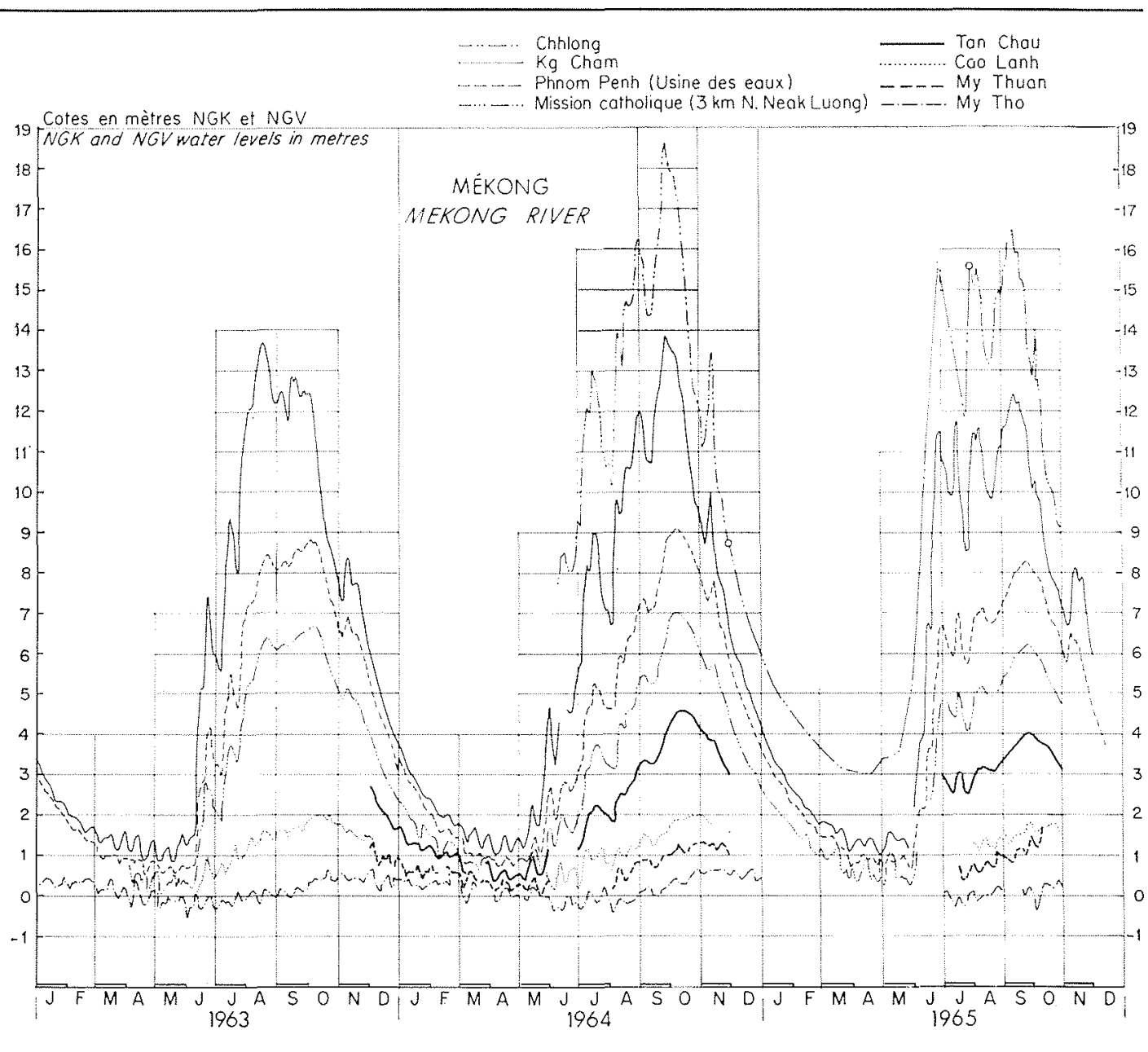




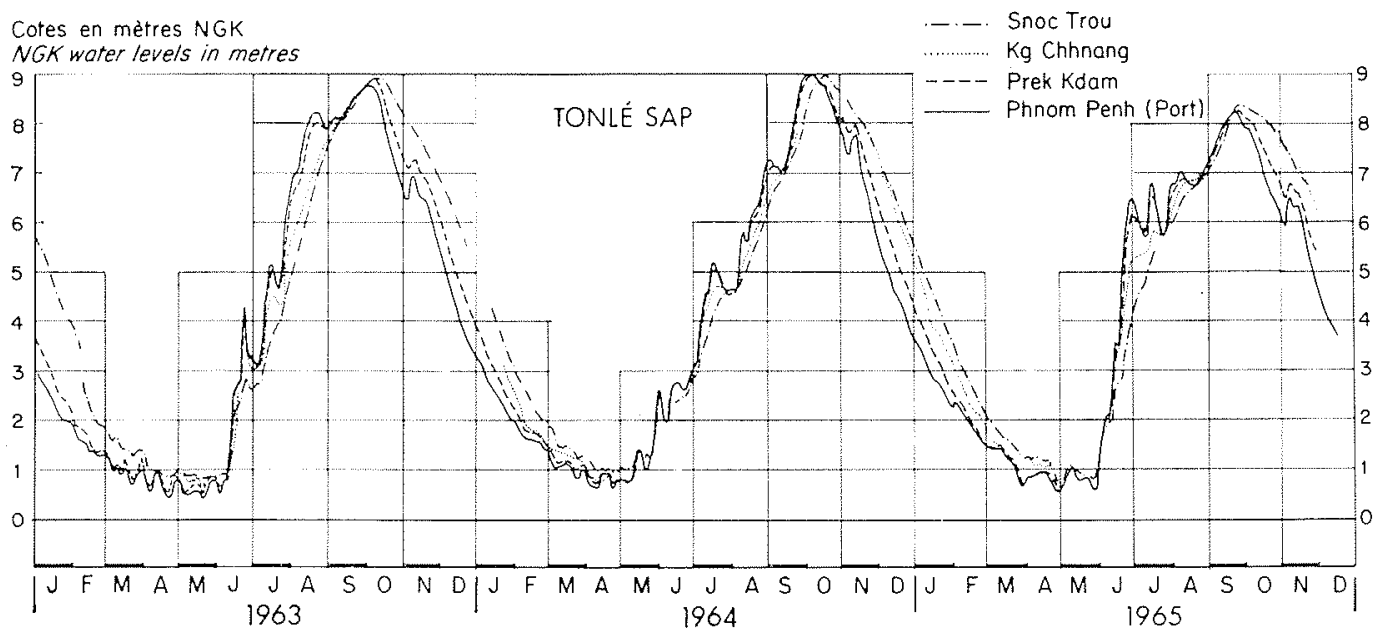

F/ Hydrogrammes des erues. Flood hydrographs.
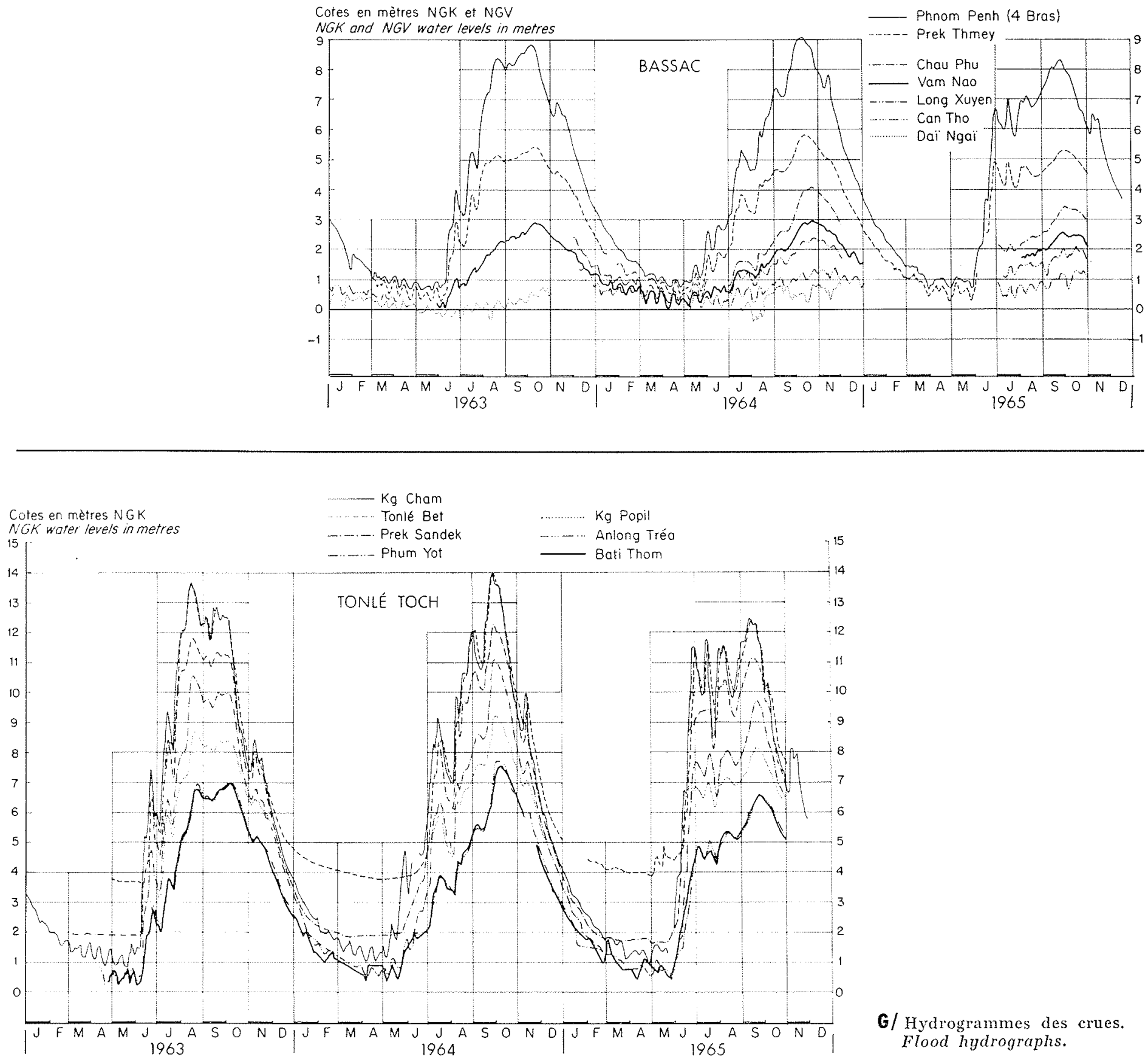

G/ Hydrogrammes des crues. Flood hydrographs. 
le Bassac, dont la pente moyenne est de $3,5.10^{-5}$, incapables de véhiculer le volume de la crue, le laisse acheminer vers l'aval en nappes latérales aux fleuves dont la destinée finale est variable:

- celle située à l'ouest du Bassac, revient soit au Bassac, soit au golfe du Siam, par les canaux et la plaine du Trans Bassac Vietnamien;

- celle comprise entre Bassac et Méliong, revient surtout au Bassac (point d'accumulation de Chauphu; bretelle du Vam Nao; déversement sud du Cis Bassac Vietnamien);

celle à l'est du Mékong revient, en partie, au Mékong, à l'extrême aval; l'autre partic, après son accumulation dans la plaine des Joncs, est lentement draînée par le Vaïco Occidental.

Dans toute section du Cis Bassac, normale à l'écoulement général, depuis Phnom Penh jusqu'au Vam Nao, le Bassac est toujours plus bas que le Mékong (près d'un mètre, au maximum). Le Mékong est donc le point haut du système, la crête faitière nourricière : il distribue l'eau à travers ses rives. Cette eau est recueillie par une succession de drains naturels (Loeuk Dek, Bassac, rivière de Vam Nao, Vaïco Occidental); ils aboutissent à l'aval, soit dans les deux fleuves principaux, soit directement à la mer (golfe du Siam); enfin, des drains ultimes constituent de véritables drains de pieds: à l'ouest du Delta, la rivière de Takéo, grossie de la rivière de Chaudoc; le Vaïco Oriental, à l'est.

On a donc une descente à la mer, par paliers intermédiaires.

Les enregistrements sont progressifs dans les grands axes hydrauliques; dans la zone inondée, certains déclenchent brusquement, passent par un maximum, puis cessent brutalement.

Ces discontinuités correspondent à la submersion, ou au dénoiement de seuils naturels qui contrôlent l'écoulement; on peut ainsi définir la cote de ces seuils, éléments très importants de l'écoulement en plaine, done du réglage du modile mathématique.

Cette détermination hydraulique de leur cote est mieux adaptée que celle provenant des relevés topographiques des terrains.

Les relevés, à grande échelle, ne sont que l'exception. Et même, si ces derniers, localement, peuvent ètre très bons, on n'est pas sûr que cet endroit commande précisément l'écoulement.

Enfin, ces seuils peurent eux-mêmes être modifiés par le paysan : il édifie des barrages en terre qui lui permettent de contrôler l'arrivée de la crue.
Autrement dit, leurs cotes varient d'une année à l'autre et les déclenchements observés ne se produisent pas, à la même cote, chaque année (Cis Bassac Cambodgien).

La marée est semi-diurne aux embouchures du Mékong et du Bassac : son amplitude maximale à Saigon dépasse $3 \mathrm{~m}$.

Elle remonte à l'intérieur, d'autant plus profondément que l'on est aux basses eaux et d'autant plus aisément que le débit de la rivière correspondante est petit.

Cette amplitude maximale décroît de la mer vers l'intérieur :

\begin{tabular}{|c|c|c|c|c|c|c|}
\hline MÉKONG & $\begin{array}{l}\text { Mer } \\
\mathrm{km} 0\end{array}$ & $\begin{array}{l}\text { Mytho } \\
\mathrm{km} 51 \\
2,65 \mathrm{~m}\end{array}$ & $\begin{array}{c}\text { Tan } \\
\text { chau } \\
\operatorname{km} 225 \\
1,09 \mathrm{~m}\end{array}$ & $\begin{array}{c}\text { Neak } \\
\text { Luong } \\
\text { km } 227 \\
0,80 \mathrm{~m}\end{array}$ & $\begin{array}{c}\mathrm{C} 15 \\
\mathrm{~km} 303 \\
0,50 \mathrm{~m}\end{array}$ & $\begin{array}{l}\text { Phnom } \\
\text { Penh } \\
\text { km } 331\end{array}$ \\
\hline BASSAC & $\begin{array}{l}\text { Mer } \\
\operatorname{lrm} 0\end{array}$ & $\begin{array}{l}\text { Daingaï } \\
\mathrm{km} 43 \\
3,19 \mathrm{~m}\end{array}$ & $\begin{array}{l}\text { Cantho } \\
\operatorname{km} 88 \\
2,37 \mathrm{~m}\end{array}$ & $\begin{array}{c}\text { Chau- } \\
\text { phu } \\
\lim 195 \\
1,13 \mathrm{~m}\end{array}$ & $\begin{array}{c}\text { Prek } \\
\text { Thmey } \\
\text { km } 245 \\
0,30 \mathrm{~m}\end{array}$ & $\begin{array}{c}\text { Phnom } \\
\text { Penl } \\
\text { km } 310\end{array}$ \\
\hline
\end{tabular}

L'amortissement de l'amplitude est le même dans le Mékong et dans le Bassac, jusqu'à environ $200 \mathrm{~km}$ de la mer; au-delà, la marée s'atténue beaucoup plus vite dans le Bassac : cet évanouissement de la marée est, très vraisemblablement, dû à la large zone inondée qui se trouve à l'amont de Chaudoc, en rive droite du Bassac; celte région d'Angkor Borey, toujours inondée, même aux basses eaux, amortit la marée.

On pourrait aussi dire parallèlement que la marée à Phnom Penh est transmise principalement par le Mékong: sa valeur extrapolée à Phnom Penh (km 331) donne bien $0,20 \mathrm{~m}$, valeur connue de l'amplitude maximale dans ce port fluvial.

Les enregistrements limnigraphiques donnent également les vitesses de propagation de la marée; mais les résultats sont plus incertains, car le facteur temps est en général moins bien connu.

Dans le Bassac, la vitesse de propagation de la marée semble avoir une valeur constante de $23 \mathrm{~km} / \mathrm{h}$ : par suite, la marée transmise par le Bassac, arrive à Phnom Penh plus de $13 \mathrm{~h}$ après s'être engagée dans l'estuaire de ce fleuve.

Dans le Mékong, la vitesse moyenne de la marée paraît plus variable: la valeur moyenne est de $28 \mathrm{~km} /$, c'est-à-dire légèrement supérieure à celle

\section{MÉKKONG :}

Durée moyenne de la propagation de la marée. . Vitesse moyenne horaire de cette proparation.

\section{BASSAC :}

Durée moyenne de la propagation de la marée. Vitesse moyenne horaire de cette propagation.

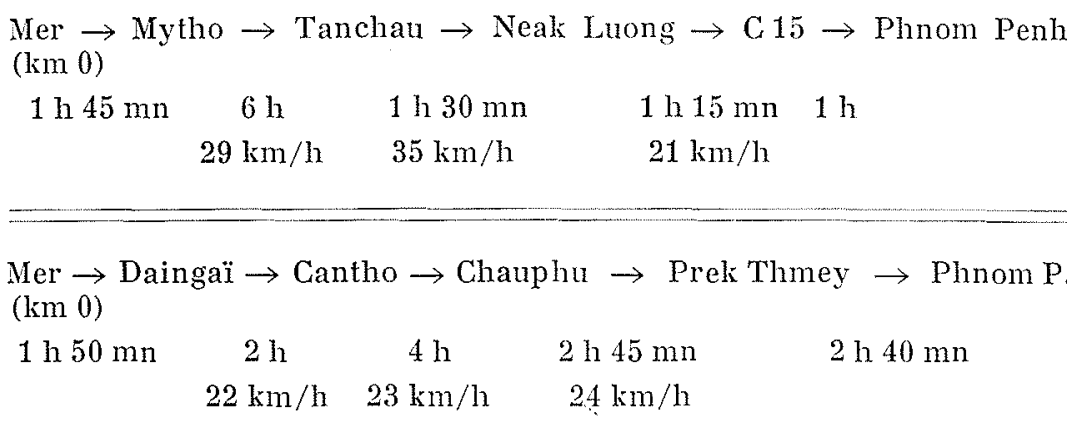


observée dans le Bassac; la marée transmise par le Mékong arrive donc $11 \mathrm{~h} 30$ après son entrée dans l'estuaire de ce fleuve.

En conclusion, la vitesse moyenne de propagation de la marée dans ces deux fleuves se situe aux environs de $25 \mathrm{~km} / \mathrm{h}$, soit $7 \mathrm{~m} / \mathrm{s}$ environ.

La même étude peut être répétée pour les deux Vaïcos.

La marée s'amortit beaucoup plus vite dans le Vaïco Occidental que dans le Vaïco Oriental; car, à l'amont de Tan An, jusqu'aux abords de Svayrieng, s'étend la marécageuse Plaine des Joncs; et, comme la marée du Bassac dans la région d'Angkor Borey, la marée du Vaïco Occidental se dilue dans la Plaine des Jones.

Quant au Vaïco Oriental, la marée y est encore très sensible à Kompong Trach, soit à $240 \mathrm{~km}$ de la mer : l'amplitude de $0,98 \mathrm{~m}$ est du même ordre de grandeur que celles constatées pour la même distance, dans le cas du Mékong $(1,01 \mathrm{~m}$ par interpolation entre Tanchan et Neak Luong).

Les enregistrements relevés sur le Vaïco Oriental peuvent permettre également d'avoir une valeur de la vitesse moyenne de propagation.

On retrouve très bien l'ordre de grandeur des vitesses de marée trouvées dans le cas du Mékong et du Bassac (la valeur moyenne était de $25 \mathrm{~km} / \mathrm{h}$ ). On trouve, en effet, pour le Vaïco Oriental, $25,8 \mathrm{~km} / \mathrm{h}$.

Toutes ces déterminations des célérités de la marée aboutissent finalement à une même moyenne de $25 \mathrm{~km} / \mathrm{h}$, soit environ $7 \mathrm{~m}$ par seconde.

\subsection{Mesure des débits.}

Au cours de ces trois campagnes, 1228 mesures de débits ont eu lieu.

EIles ont intéressé :

- les grands fleuves : Mékong, Bassac, 'Tonlé Sap, Vam Nao;

- les rivières secondaires du Delta, en général by-pass naturels, dont l'origine partielle ou totale peut être un ancien bras mort: Muk Kompul, Tonlé Toch, Loeuk Dek; rivières de Chaudoc, de Takéo, de Chomoï, de Hong Ngu;

- les canaux (Vietnam);

- les affluents indépendants du Mékong: Prek Thnot, Stung Slakou, qui ont un bassin versant propre;

- les rivières à caractère hybride, ayant leur propre bassin, mais alimentées par l'inondation du Mékong pendant une plus ou moins grande partie de l'année.

Les deux Vaïcos, Oriental et Occidental, sont de ce dernier type :

- Le Vaico Oriental coule du nord-ouest au sudest, au pied de la dernière avancée de la montagne, avant le Delta:

Bassin propre: tous les écoulements provenant de cette avancée montagneuse.

Alimentation par le Mékong : lorsque la crue du Mékong amont arrive, une faible partie des eaux de débordement en rive gauche est détournée vers le Vaïco supérieur, pour constituer « la crue régulière » annuelle de la rivière.

- Le Vaïco Occidental est compris entre le Mékong aval et le Vaïco Oriental.

Bassin propre: négligeable;
Alimentation par le Mékong : très importante; Ie Vaïco draîne la vaste Plaine des Jones, elle-même inondée par le Mékong aval.

Les débits mesurés couvrent une gamme très étendue de valeurs, depuis $2,8 \mathrm{~m}^{3} / \mathrm{s}$ jusgu'à $34640 \mathrm{~m}^{3} / \mathrm{s}$.

- Si, en général, dans le Delta Cambodgien, les débits restent constanls pendant le jour, il n'en est pas de même au Vietnam, où le Delta avance en coin entre la mer de Chine el le golfe du Siam.

L'influence de la marée produit très souvent l'inversion des courants, visible très loin, en amont : ainsi l'observe-t-on à Kompong Kraissaing, sur le Vaïco Oriental, c'est-à-dire à plus de $260 \mathrm{~km}$ de la mer.

L'énormité des débits, circulant dans les fleuves ou dans la zone inondée, est plus liée à celle des sections monillées qu'à la grandeur des vitesses.

L'inondation est plutot statique, non dynamique, exception faite localement, dans le temps et l'espace où enclenchent des brêches, ou des seuils alimentant des zones en contrebas. Si l'inondation avait un caractère plus dynamique, ce Delta n'aurait pas la densité de population qu'il a.

$\mathrm{Et}$, en effet, les vitesses moyennes mesurées dans les grands fleuves n'ont pas été très élevées:

\section{cambodge :}

Mékong amont. . . 0 0,14 m/s $<\mathrm{V}_{\mathrm{MI}}<1,66 \mathrm{~m} / \mathrm{s}$

- Mékong aval. . . . . 0,38 m/s $<\mathrm{V}_{\mathrm{M}}<1,22 \mathrm{~m} / \mathrm{s}$

- Bassac. . . . . . $0,06 \mathrm{~m} / \mathrm{s}<\mathrm{V}_{\mathrm{MI}}<1,18 \mathrm{~m} / \mathrm{s}$

_. Tonlé Sap. ...... $0,19 \mathrm{~m} / \mathrm{s}<\mathrm{V}_{\mathrm{M}}<1,15 \mathrm{~m} / \mathrm{s}$

\section{Vietnam :}

- Mékong. . . . . . . $0,98 \mathrm{~m} / \mathrm{s}<\mathrm{V}_{\mathrm{M}}<1,47 \mathrm{~m} / \mathrm{s}$

- Bassac. . . . . . . 0, 0,33 m/s $<\mathrm{V}_{\mathrm{M}}<1,42 \mathrm{~m} / \mathrm{s}$

- Vam Nao....... $0,98 \mathrm{~m} / \mathrm{s}<V_{\mathrm{M}}<1,14 \mathrm{~m} / \mathrm{s}$

A la limite, la vitesse moyenne la plus élevée qui ait été mesurée a été :

- en 1963:

$1,60 \mathrm{~m} / \mathrm{s}$ (Tonlé Sap) au Cambodge,

$1,90 \mathrm{~m} / \mathrm{s}$ (Orifice roulier : route Tinh Bien - Chaudoc) au Vietnam;

- en 1964:

$1,97 \mathrm{~m} / \mathrm{s}$ (Orifice routier : parcelle $\mathrm{km} 54$ ) au Cambodge,

$1,10 \mathrm{~m} / \mathrm{s}$ (Orifice routier : Caïvon) au Vietnam.

$$
\text { - en 1965: }
$$

$1,18 \mathrm{~m} / \mathrm{s}$ (Bassac) au Cambodge,

$0,93 \mathrm{~m} / \mathrm{s}$ (Canal d'O Mon) au Vietnam.

Une vitesse moyenne de $2 \mathrm{~m} / \mathrm{s}$ aurait donc constitué un maximum : il est vrai que les crues 19631964-1965 n'ont pas été fortes; d'autre part, les vitesses moyennes les plus élevées ont été obtenues au droit d'obstacles locaux, orifices routiers où se produisent des concentrations artificielles de débit.

II ne semble pas toutefois que l'augmentation de la vitesse moyenne, à l'occasion d'une crue forte, soit importante : l'accroissement correspondant de débit proviendrait plutôt de l'accroissement de la section mouillée par sa partie haute où précisément les vitesses locales sont les plus élevées.

Quant aux régimes soumis à la marée, les vitesses moyennes maximales, données dans le tableau de la page suivante, ont été mesurées dans les Vaïcos. 


\begin{tabular}{|c|c|c|}
\hline $\begin{array}{l}\text { Vaico Oriental : } \\
\text { _- vitesse moyenne. . . . . . . . } \\
\text { _ débit instantané maximal..... }\end{array}$ & $\begin{array}{c}\text { Go Dau } \mathrm{Ha}(150 \mathrm{~km} \text { de la mer) } \\
-0,59 \mathrm{~m} / \mathrm{s}<\mathrm{V}_{M}<0,67 \mathrm{~m} / \mathrm{s} \\
\text { vers le nord vers le sud } \\
926 \mathrm{~m}^{3} / \mathrm{s}\end{array}$ & $\begin{array}{l}\text { Ben Luc }(61 \mathrm{~km} \text { de la mer }) \\
-1,06 \mathrm{~m} / \mathrm{s}<\mathrm{V}_{\mathrm{M}}<1,16 \mathrm{~m} / \mathrm{s} \\
\text { vers Ie nord vers le sud } \\
1720 \mathrm{~m}^{3} / \mathrm{s}\end{array}$ \\
\hline $\begin{array}{c}\text { Vaico Occidental : } \\
\text { _ vitesse moyenne. . . . . . . } \\
\text { _ débit instantané maximal..... }\end{array}$ & & $\begin{array}{c}\text { Tan An } \\
-1,06 \mathrm{~m} / \mathrm{s}<\mathrm{V}_{\mathrm{M}}<1,16 \mathrm{~m} / \mathrm{s} \\
\text { vers le nord } \text { vers le sud } \\
2224 \mathrm{~m}^{3} / \mathrm{s}\end{array}$ \\
\hline
\end{tabular}

La vitesse vers le nord, c'est-à-dire à contrepente, est bien inférieure à la vitesse sud. Mais Ia différence est faible: voilà pourquoi ces deux rivières ont de la peine à véhiculer leur débit propre jusqu'à la mer.

Ce tableau montre, d'autre part, que le débil instantané n'a aucun rapport avec le débit moyen quotidien, correspondant au débit propre de la rivière.

Les deux rivières vélhiculent des débits extrêmes analogues, correspondant à des gammes semblables de vitesse: ces deux rivières ont donc une grande analogie, au moins dans leur cours aval, que traduit leur vocable commun de Vaïco.

Les mesures de débit effectuées sur les grands fleuves du Delta n'ont pas été continues et n'ont eu lieu qu'à certaines époques; cependant, le modèle mathématique, réglé sur les crues 1963,1964 , permet de suivre l'évolution des débits tout au long de la crue et tout an long des fleuves.

On aboutit de la sorte aux maximaux suivants $\left(\right.$ en $\left.\mathrm{m}^{3} / \mathrm{s}\right)$

Mékong :

$\begin{array}{rcrcc} & \begin{array}{c}\text { Kompong } \\ \text { Cham }\end{array} & \begin{array}{c}\text { Phnom } \\ \text { Penh }\end{array} & \text { Tanchau } & \text { Mythuan } \\ -1963 & 44844 & 39835 & 24210 & 17339 \\ -1964 & 47146 & 40177 & 25329 & 18523\end{array}$

BASSAC :

$\begin{array}{cccc} & \text { Phnom Penh } & \text { Chaudoc } & \text { Cantho } \\ -1963 & 6134 & 6179 & 13110 \\ -1964 & 6560 & 6336 & 13048\end{array}$

\begin{tabular}{ccc} 
Tonté SAP : & \multicolumn{2}{c}{ Prek Kdam } \\
-1963 & Crue & Décrue \\
-1964 & 9195 & 9100 \\
-1964 & 7743 & 8291
\end{tabular}

VAM NAO :

$\begin{array}{ll}-1963 & 7199 \\ -1964 & 7546\end{array}$

Le Mékong s'appauvrit donc continûment d'amont en aval et inversement, le Bassac s'enrichit, surtout à l'extrême aval, jusqu'à véhiculer un débit comparable à celui du Mékong.

Le modèle mathématique donne l'évolution des crues 1963,1964 et du 1 er juillet au 30 novembre : il peut donc fournir pour ces deux années, les volu-

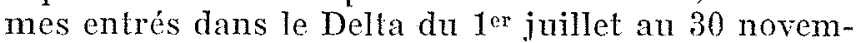
bre (débit à Kompong Cham et by-pass latéral en rive gauche).
Volume annuel entrant dans le Delta:

- 1963 : 418 milliards de $\mathrm{m}^{3}$;

- 1964 : 419 milliards de $\mathrm{m}^{3}$ (414 milliards de $\mathrm{m}^{3}$, d'après l'Annuaire hydrologique du Comité du Mékong, 1964).

Le volume annuel du Mékong, à son entrée dans le Delta, esi environ huit fois celui du Rhône à son extrême aval.

\subsection{Mesures de salinité.}

Les mesures ont eu lien en vingt et une stations réparties dans le Delta Vietnamien depuis Rachgia jusqu’à Saigon.

Par suite des circonstances, elles n'ont pu avoir lieu aussi près de la bordure maritime qu'il aurait été souhaitable.

Les valeurs importantes de la salinité se trouvent :

- dans le sud de la presqu'île de Caman : cette langue de terre baignée par la mer sur deux côtés est, en outre, la zone du Delta la plus éloignée de l'eau douce apportée par le Mékong, directement ou indirectement;

- au nord-est du Vaïco Occidental : la mer pénètre en coin dans les terres par le large chenal de la rivière de Saigon; et on s'éloigne également de l'apport d'eau donce du Mékong.

Par contre, le front salin est le plus repoussé vers la mer dans le Cis Bassac, étroitement enserré entre Bassac et Mékong, puissants génératcurs d'eau douce.

La valeur maximale de la salinité, qu'on ait mesurée, a été de $35,10 \mathrm{~g} / 1$ (les archives domment $36,7 \mathrm{~g} / 1$ ).

Il n'y a pas été observé de coin salé : les prélèvements de salinité effectués sur une même verticale en différentes sections du Delta n'ont pas décelé d'interface salin; le mélange est dans la plupart des cas d'autant plus homogène que la concentration est forte (presqu'île de Camau).

\subsection{Mesures de turbidité.}

La turbidité du Mékong, et a fortiori de la zone inondée, est faible : $0,75 \mathrm{~g} / 1$ au maximum.

Il suffit de Iui comparer les valeurs maximales atteintes dans d'autres fleuves :

$12 \mathrm{~g} / \mathrm{l}$ dans le Rhône à l'aval d'Avignon;

$70 \mathrm{~g} / 1$ dans le Sefid Roud (Iran);

$300 \mathrm{~g} / \mathrm{l}$ dans le Fleuve Jaune. 
La crue brutale de 1964 a provoqué une crue solide, beaucoup plus concentrée, sans que le maximum soit supérieur aux valeurs des autres années 1963-1965.

Comme il est général, la crue solide a précédé la crue liquide de près de trois mois : son démarrage est dû à l'entraînement des désagrégations de saison sèche par les premières pluies.

Aux Quatre-Bras, en 1964, les mesures permettent une évaluation du tonnage en suspension dans le Méliong amont:

94,3 millions de tonnes pour 1964.

Le Rhône, à l'amont de son Delta, en a véhiculé 17,1 millions de tonnes pendant une année (septembre 1956 - septembre 1957).

Le tonnage annuel Mékong est done 5,5 fois celui du Lonnage Rhône; ce rapport est assez voisin de celui des volumes d'eau annuels (huit fois).

\subsection{Mesures météorologiques.}

Deux stations fondamentales ont été installées au centre de chacun des Deltas:

- Delta Cambodgien : Prey Veng;

-- Delta Vietnamien : Cao Lanh.

Elles ont permis chacune les mesures:

- du vent:

- un anémomètre enregistreur,

- une girouetle enregistreuse;

- de la pluie:

- un pluviographe,

- un pluviomètre,

- un pluviomètre association;

- de l'ensoleillement : un solarimètre;

- de la température:

- un thermographe;

- un thermomètre à maximum-minimum;

- de la pression: un barographe;

- de l'évaporation :

- un hygrographe;

- un bac évaporation type Weather Bureau
« $A$ » muni d'un anémomètre à $50 \mathrm{~cm}$ audessus du bac;

- un évaporomètre Piche (sous abri);

- un psychromètre (sous abri).

\begin{tabular}{|c|c|c|c|c|c|c|}
\hline \multicolumn{7}{|c|}{$\begin{array}{c}\text { Valeurs extrêmes quotidiennes } \\
\text { CAO LANH (Vietnam) }\end{array}$} \\
\hline \multirow[b]{2}{*}{$\begin{array}{c}\text { Température } \\
\left({ }^{\circ} \mathrm{C}\right) \ldots \ldots\end{array}$} & \multicolumn{2}{|c|}{$\begin{array}{c}1963 \\
\text { à partir du } \\
1-6-63\end{array}$} & \multicolumn{2}{|c|}{1964} & \multicolumn{2}{|c|}{$\begin{array}{c}1965 \\
\text { jusqu'au } \\
31-10-65\end{array}$} \\
\hline & $24^{\circ} 4$ & $29^{\circ} 3$ & $22^{\circ} 1$ & $33^{\circ} 9$ & $19^{\circ} 7$ & $31^{\circ} 6$ \\
\hline $\begin{array}{l}\text { Evaporation } \\
\text { bac }(\mathrm{mm} / \mathrm{j})\end{array}$ & 0,9 & 10,8 & 0,5 & 8,9 & 0,4 & 9,4 \\
\hline $\begin{array}{l}\text { Humidité } \\
\text { relative. . }\end{array}$ & 72 & 96 & 50 & 97,5 & 52 & 96 \\
\hline $\begin{array}{l}\text { Pluie totale } \\
(\mathrm{mm}) \ldots \ldots\end{array}$ & \multicolumn{2}{|c|}{1139} & \multicolumn{2}{|c|}{1524} & \multicolumn{2}{|c|}{1484} \\
\hline
\end{tabular}

\begin{tabular}{|c|c|c|c|c|c|c|}
\hline \multirow[b]{3}{*}{$\begin{array}{l}\text { Température } \\
\left({ }^{\circ} \mathrm{C}\right) . \ldots \ldots\end{array}$} & \multicolumn{6}{|c|}{$\begin{array}{l}\text { urs extrêmes quotidiennes } \\
\text { REY VENG (Cambodge) }\end{array}$} \\
\hline & \multicolumn{2}{|c|}{$\frac{1963}{\text { a partir du }}$} & \multicolumn{2}{|c|}{1964} & \multicolumn{2}{|c|}{$\begin{array}{c}1965 \\
\text { juscyu'au } \\
31-10-65\end{array}$} \\
\hline & $23^{\circ} 4$ & $31^{\circ} 6$ & $22^{\circ} 1$ & $31^{\circ} 5$ & $21^{\circ} 4$ & $30^{\circ} 9$ \\
\hline $\begin{array}{l}\text { Evaporation } \\
\text { bac }(\mathrm{mm} / \mathrm{j}) \text {. }\end{array}$ & 1,0 & 8,6 & 0,3 & 9,9 & 0,2 & 11,4 \\
\hline $\begin{array}{l}\text { Humidité } \\
\text { relative. . }\end{array}$ & 55 & 94 & 47 & 99 & 47 & 97 \\
\hline $\begin{array}{l}\text { Pluie totale } \\
(\mathrm{mm}) \ldots \ldots\end{array}$ & \multicolumn{2}{|c|}{1421} & \multicolumn{2}{|c|}{1692} & \multicolumn{2}{|c|}{1574} \\
\hline
\end{tabular}

(A suivre.) 


\section{Abstract \\ A mathematical model of the Mekong delta \\ by D. Zanobetti * and H. Lorgeré * *}

\section{1st PART}

\section{The problem.} ditions.

The Mekong runs through several countries, governing both their economic development and human living con-

Thanks to the availability of powerful modern facilities and such important international liaison organisations as UNESCO's Mekong Committee, development of the Mekong could be visualised in terms of major polyvalent development units concerning many of the riparian countries. One of the first such units is the Tonle Sap dam. Under present conditions, when the Mekong flood reaches the capital of Cambodia, Phnom Penh, it fills up the gigantic natural reservoir of the Grand Lac through the Tonle Sap river, and when the flood recedes, the Grand Lac drains back into the Mekong by the same way. This quite appreciable regulating effect, however, cannot cope with floods rising or falling excessively fast, rising too high or not falling low enough; in order to remedy this shortcoming, it is planned to build a dam on the river Tonle Sap linking the Grand Lac and the Mekong, which will control the filling and emptying of the Grand Lac. Before embarking upon the construction of such a dam, however, it was considered vital to determine and provide a numerical illustration of its effects on various factors governing hydraulic conditions in the Delta.

\section{The mathematical model.}

A mathematical model was necessary to investigate the hydraulic effects of the dam, with the following requirements:

(i) That is should be able to represent natural flood propagation in the Delta;

(ii) That it should feature the dam;

(iii) That it should enable comparison of results with and without the dam.

It was decided to use a mathematical model for this hydraulic investigation because of the very large area to be featured $(50,000 \mathrm{sq} . \mathrm{kms})$ and the tremendous range of the natural phenomena involved (velocities varying between a few millimetres/sec, and 2.5 metres/sec, depths from a few decimetres up to thirty metres or so, tide structures varying with shore position, etc.).

The study was financed by the United Nations Special Fund and the riparian countries, managed by UNESCO, and carried out by Société Grenobloise d'Etudes et d'Applications Hydrauliques (SOGREAH). It covers the Mekong Delta and concerns two countries: Cambodia and Vietnam.

\footnotetext{
* Professeur à l'Université de Bologne, ancien directeur de Ia Division des études, de la recherche et de l'enseignement technique de l'Organisation des Nations Unies pour l'Education, la Science et la Culture (UNESCO) et directeur du projet du modèle mathématique du Delta du Mékong;

* Chef adjoint du Service hydroélectrique a la Société Grenobloise d'Etudes et d'Applieations Hydrauliques (SOGREAH) de Grenoble et ingénieur en chef du projet du modèle mathématique du Delta du Méliong.
} 